\title{
Risco cardiovascular em profissionais de saúde de serviços de atendimento pré-hospitalar*
}

\author{
CARDIOVASCULAR RISK AMONG HEALTH PROFESSIONALS WORKING IN PRE- \\ HOSPITAL CARE SERVICES
}

\section{RIESGO CARDIOVASCULAR EN PROFESIONALES DE SALUD DE SERVICIOS DE ATENCIÓN PREHOSPITALARIA}

\author{
Luciane Cavagioni ${ }^{1}$, Angela Maria Geraldo Pierin ${ }^{2}$
}

\begin{abstract}
RESUMO
Avaliar os fatores de risco cardiovascular, com ênfase na hipertensão, e estratificálos de acordo com o Escore de Risco de Framingham (ERF). Estudo com 154 profissionais que atuavam em atendimento pré-hospitalar na cidade de São Paulo e rodovia $\mathrm{Br}-116$. Foi considerado significante $o$ valor de $p<0,05$. A prevalência de hipertensão foi de $33 \%$, sendo que $20,1 \%$ eram tabagistas, $47 \%$ ingeriam bebidas alcoólicas, $64 \%$ eram sedentários, $66 \%$ apresentaram obesidade/sobrepeso e $70 \%$ cintura abdominal alterada, glicemia $\geq 110 \mathrm{mg} /$ $\mathrm{dL}-11 \%$, colesterol total $\geq 200 \mathrm{mg} / \mathrm{dL}-36 \%$, LDL-c $\geq 130 \mathrm{mg} / \mathrm{dL}-\quad 33 \%$, HDL-c $<60 \mathrm{mg} /$ dL- $89 \%$, triglicérides $\geq 150 \mathrm{mg} / \mathrm{dL}-30 \%$ e proteína $C$ reativa $\geq 0,5 \mathrm{mg} / \mathrm{dL}-16 \%$. O ERF foi médio em $10,3 \%$ e alto em $1,3 \%$. Na análise de regressão logística verificouse que a hipertensão associou-se com as variáveis: HDL-c (odds ratio: 0,257) e ERF (odds ratio: 23,159). Houve forte associação entre ERF e hipertensão. Os dados chamam a atenção, por se tratar principalmente de profissionais da área da saúde relativamente jovens.
\end{abstract}

\section{DESCRITORES}

Hipertensão

Fatores de risco

Pessoal de saúde

\begin{abstract}
The objective of this study was to assess the cardiovascular risk factors among health professionals, particularly hypertension, and stratify them according to the Framingham Risk Score (FRS). The participants were 154 professionals working in pre-hospital care in São Paulo, Brazil, and on the $\mathrm{Br}-116$ highway. Values were considered significant for $\mathrm{p}<0.05$. The prevalence of hypertension was 33\%, 20.1\% were smokers, $47 \%$ consumed alcoholic beverages, $64 \%$ were sedentary, $66 \%$ were obese/overweight and $70 \%$ had an altered abdominal circumference. In terms of laboratory values: glucose $\geq 110 \mathrm{mg} / \mathrm{dL}-$ $11 \%$, total cholesterol $\geq 200 \mathrm{mg} / \mathrm{dL}-36 \%$, LDL-c $\geq 130 \mathrm{mg} / \mathrm{dL}-33 \%$, HDL-c $<60 \mathrm{mg} / \mathrm{dL}-$ $89 \%$, triglycerides $\geq 150 \mathrm{mg} / \mathrm{dL}-30 \%$ and C reactive protein $\geq 0.5 \mathrm{mg} / \mathrm{dL}-16 \%$. The FRS was average in $10.3 \%$ and high in $1.3 \%$. In logistic regression analysis, it was verified that hypertension was associated with: HDL-c (odds ratio: 0.257,) and FRS (odds ratio: 23.159). There was strong correlation between hypertension and FRS. Data are noteworthy, as this is a relatively young sample of health professionals.
\end{abstract}

\section{DESCRIPTORS \\ Hypertension \\ Risk factors \\ Health personnel}

\section{RESUMEN}

Evaluar los factores de riesgo cardiovascular con énfasis en hipertensión y estratificarlos de acuerdo a Score de Riesgo de Framingham (ERF). Estudio con 154 profesionales con actuación en atención prehospitalaria en ciudad de São Paulo y ruta $\mathrm{Br}-116$. Se consideró significativo el valor $\mathrm{p}<0,05$. La prevalencia de hipertensión fue $33 \%, 20,1 \%$ eran fumadores, $47 \%$ ingería bebidas alcohólicas, $64 \%$ sedentarios, $66 \%$ con obesidad/sobrepeso, $70 \%$ con cintura abdominal alterada, glucemia $\geq 110 \mathrm{mg} /$ dl- $11 \%$, colesterol total $\geq 200 \mathrm{mg} / \mathrm{dl}-30 \%$, LDL-c $\geq 130 \mathrm{mg} / \mathrm{dl}-33 \%$, HDL-c $<60 \mathrm{mg} / \mathrm{dl}-$ $89 \%$, triglicéridos $\geq 150 \mathrm{mg} / \mathrm{dl}-30 \%$ y proteína C reactiva $\geq 0,5 \mathrm{mg} / \mathrm{dl}-16 \%$. El ERF fue medio en $10,3 \%$ y alto en 1,3\%. En análisis de regresión logística se verificó que la hipertensión se asociaba a las variables: HDL-c (odds ratio: 0,257), y ERF (odds ratio: 23,159$)$. Hubo fuerte asociación entre ERF e hipertensión. Los datos llaman la atención por tratarse principalmente de profesionales del área de la salud relativamente jóvenes.

\section{DESCRIPTORES}

Hipertensión

Factores de riesgo

Personal de salud

\footnotetext{
* Extraído da tese "Influência do plantão de 24 horas sobre a pressão arterial e o perfil de risco cardiovascular em profissionais da área da saúde que atuam em serviços de atendimento pré-hospitalar", Programa de Pós-Graduação. ${ }^{1}$ Enfermagem na Saúde do Adulto da Escola de Enfermagem da Universidade de São Paulo. São Paulo, 2010. Enfermeira. Doutora em Enfermagem pelo Programa de Pós-Graduação Enfermagem na Saúde do Adulto da Escola de Enfermagem da Universidade de São Paulo. São Paulo, SP Brasil. Iuciane.cavagioni@gmail.com ${ }^{2}$ Professora Titular do Departamento de Enfermagem Médico-Cirúrgica da Escola de Enfermagem da Universidade de São Paulo. São Paulo, SP. Brasil. pierin@usp.br
} 


\section{INTRODUÇÃO}

Segundo a World Health Organization as doenças cardiovasculares são responsáveis por, aproximadamente, $30 \%$ das mortes em todo o mundo e nos países desenvolvidos $80 \%$ das mortes podem ser atribuídas às doenças cardiovasculares ${ }^{(1)}$. Estas apresentam uma etiologia multifatorial decorrente de hábitos e estilos de vida inadequados como o tabagismo, ingestão de bebidas alcoólicas, estresse físico e mental, obesidade e sedentarismo. Esses estilos de vida contribuem para o desenvolvimento de aterosclerose e hipertensão arterial que são fatores responsáveis pelo desenvolvimento das doenças cardiovasculares. Dados do Ministério da Saúde revelam que as doenças cardiovasculares foram as principais causas de mortalidade no ano de 2007 com 30\% dos óbitos ${ }^{(2)}$. Em razão de sua alta prevalência, cronicidade, baixo controle e elevado custo sócio-financeiro, a hipertensão arterial destaca-se dentre os fatores de risco cardiovascular. Estima-se que há cerca de 1 bilhão de indivíduos hipertensos no mundo, sendo a hipertensão responsável por, aproximadamente, 7,1 milhões de óbitos por ano ${ }^{(3)}$.

Nas últimas décadas outros fatores de risco emergentes foram o sobrepeso/obesidade e o sedentarismo. As autoridades sanitárias e os estudiosos consideram o excesso de peso e o sedentarismo como uma pandemia havendo uma correlação positiva entre o excesso de peso, o sedentarismo e as altas prevalências de hipertensão arterial. Corroborando com essa visão, o estudo de Framingham revelou que $70 \%$ dos novos casos de hipertensão arterial estavam relacionados diretamente ao excesso de peso ${ }^{(4)}$.

Dentro do contexto atual de prevenção cardiovascular não se deve caracterizar o indivíduo só como hipertenso, hiperlipidêmico, obeso ou diabético, mas, também, determinar seu risco cardiovascular total. Um dos estudos que mais contribuiu para essa nova visão de risco cardiovascular total foi o estudo de Framingham. Após seu término foi elaborado um escore que estima o risco cardiovascular, pela soma das características clínicas, permitindo aos profissionais de saúde que tomem melhor decisão terapêutica e, em conjunto com o paciente, elaborarem intervenções preventivas de acordo com o risco cardiovascular total encontrado e a prevenção primária das doenças cardiovasculares ${ }^{(5)}$.

Os profissionais de saúde que atuam no atendimento pré-hospitalar podem estar sujeitos aos fatores de risco cardiovascular pelo estilo de vida adotado e pelas características específicas de sua ocupação. Em nosso meio não existem registros conhecidos sobre o risco cardiovascular entre esses profissionais. Desse modo o presente estudo teve como objetivos: avaliar fatores de risco cardiovascular, com ênfase na hipertensão arterial, e estratificá-los, de acordo com o Escore de Risco de Framingham (ERF).

\section{MÉTODO}

Tratou-se de um estudo transversal com 154 profissionais da área da saúde que atuavam nos seguintes serviços de atendimento pré-hospitalar: Serviço de Atendimento Médico de Urgência - 192 (SAMU-192) e Grupo de Atendimento às Urgências - 193 (GRAU-193), ambos da cidade de São Paulo com abrangência nas quatro regiões da cidade (norte, sul, leste e oeste), e SAMU - Vale do Ribeira-SP, localizado na Rodovia BR-116. O cálculo do tamanho da amostra foi realizado para uma variável dicotômica em estudo descritivo, no qual a proporção de casos esperados foi de $20 \%$, com a largura de confiança de 0,10 (0,5 acima e 0,5 abaixo) a um nível de confiança de $99 \%$, obtendo-se amostra mínima de 130 participantes. Os critérios de inclusão foram: ser profissional da área da saúde, atuar diretamente no atendimento às vítimas e ter no mínimo três meses de atividade profissional no serviço pré-hospitalar. Os profissionais foram convidados para participar do estudo de forma aleatória, através de contato telefônico, e-mail ou pessoalmente em suas bases de trabalho. Todos assinaram o Termo de Consentimento Livre e Esclarecido, tendo sido o projeto aprovado pelo Comitê de Ética da Escola de Enfermagem da Universidade de São Paulo, sob no 742/2008.

A verificação da pressão arterial foi realizada com aparelho automático validado (OMROM HEM 705CP) no local de trabalho dos participantes, seguindo as orientações das VI Diretrizes Brasileiras de Hipertensão(6) que preconiza: três medidas; após 5 minutos de repouso; posição sentada; costas apoiadas e pernas descruzadas; uso de manguito com largura e comprimento adequados ao tamanho do braço; bexiga urinária vazia; e não ter fumado e/ou ingerido bebidas alcoólicas, café e alimentos há, pelo menos, 30 minutos. Para a análise estatística foi considerada a média das duas últimas aferições, sendo os participantes classificados como hipertensos quando a pressão sistólica fosse $\geq 140 \mathrm{mmHg}$ e/ou pressão diastólica $\geq 90 \mathrm{mmHg}$ ou referência de uso de anti-hipertensivos.

Os participantes receberam um questionário autoaplicável com as seguintes variáveis socioeconômicas: idade (em anos); categoria profissional (médico, enfermeiro e auxiliar de enfermagem); tabagismo (qualquer quantidade de cigarro diária e os que pararam há menos de 1 ano); consumo de bebidas alcoólicas (considerados bebedores todos que referiram uso independente do tipo e quantidade); atividade física (considerada regular a prática de, pelo menos, 30 minutos de atividade, de forma contínua ou acumulada, três ou mais vezes por semana); tempo de formado; carga de trabalho semanal e uso atual de medicamentos. 
O peso (em kilogramas) e a altura (em metros) foram determinados por meio de uma balança antropométrica mecânica, possibilitando o cálculo do Índice de Massa Corpórea (peso/altura2) classificado segundo os critérios da Organização Mundial da Saúde (OMS) em: saudável (<25 kg/ $\mathrm{m} 2)$, sobrepeso $(25$ a $29,9 \mathrm{~kg} / \mathrm{m} 2)$ e obeso $(\geq 30 \mathrm{~kg} / \mathrm{m2})^{(7)}$. $\mathrm{Na}$ determinação da circunferência abdominal a medida foi realizada à meia distância, entre a crista ilíaca e o rebordo costal, usando-se fita métrica inelástica ajustada ao corpo, adotando-se a classificação de acordo com os critérios da OMS (para o sexo masculino: $<94 \mathrm{~cm}$, normal; $\geq 94 \mathrm{~cm}$, risco aumentado; e $\geq 102 \mathrm{~cm}$, risco muito aumentado. Para o sexo feminino os valores foram: $<80 \mathrm{~cm}$, normal; $\geq 80 \mathrm{~cm}$, risco aumentado; $\mathrm{e} \geq 88 \mathrm{~cm}$, risco muito aumentado).

Para a coleta de exames laboratoriais usou-se o sistema vacutainer. Foi colhido sangue venoso antecubital, após 12 horas de jejum e encaminhado, logo em seguida, ao laboratório. Após a obtenção dos resultados laboratoriais os participantes foram classificados de acordo com seu perfil lipídico, glicemia e a proteína C reativa (em quartis).

Com base no escore de risco de Framingham foi calculado o risco cardiovascular global de cada indivíduo. $\mathrm{O}$ escore de risco de Framingham inclui em sua análise os seguintes fatores de risco: idade, colesterol total, HDL-c, pressão arterial, diabetes e tabagismo, com pontuação específica para cada um dos itens ${ }^{(8)}$. A soma dos pontos conferiu o escore global de cada indivíduo assim discriminado: baixo risco $\leq$ a $10 \%$; médio risco, maior que $10 \%$, porém, menor do que $20 \%$; e alto risco $\geq$ a $20 \%$.

Para a análise estatística foi usado o software Statistical Package for Social Sciences (SPSS) versão 7.5. As variáveis classificatórias descritivas são apresentadas em frequências absolutas ( $n$ ) e relativas (\%) e os dados contínuos em média e desvio padrão. Os testes Qui-quadrado e o exato de Fischer foram usados quando pertinentes. $O$ valor de $p<0,05$ foi considerado estatisticamente significante. As variáveis que apresentaram significância na análise univariada foram submetidas à regressão logística.

\section{RESULTADOS}

Foram avaliados 154 profissionais, sendo pouco mais da metade da amostra composta por enfermeiros (59\%), seguida pelos médicos (26\%) e auxiliares de enfermagem (15\%). Houve predomínio do sexo feminino (60\% vs $40 \%)$ e de adultos jovens $(40,9 \pm 7,8$ anos). A referência de familiares com hipertensão foi elevada (82,5\%), e apenas $20,1 \%$ citaram antecedentes pessoais. Em relação aos hábitos de vida, um quinto informou ser tabagista, porém a ingestão de bebidas alcoólicas foi bem mais elevada $(47,5 \%)$, o mesmo ocorrendo com a falta de atividade física regular $(65 \%)$. No tocante às características profissionais, pouco menos da metade $(43,5 \%)$ trabalhava mais que 60 horas semanais e, montante similar, $(45 \%)$ referiu dormir durante o dia quando estava no serviço de atendi- mento pré-hospitalar. A maioria possuía duas décadas de formada (74\%), trabalhava até 10 anos no serviço (70\%) e mais da metade mencionou trabalhar cansado frequentemente, ou algumas vezes $(58,8 \%)$. Os entrevistados informaram ainda que atualmente realizavam tratamento de saúde $(48 \%)$, sendo o mais apontado o tratamento antidepressivo (77\%). Verificou-se também a informação de acidentes durante o atendimento no serviço pré-hospita$\operatorname{lar}(43,5 \%)$, sendo o mais frequente lesões com material perfurocortante (33\%) (Tabela 1).

Tabela 1 - Características sóciodemográficas dos profissionais que atuam no atendimento pré-hospitalar - São Paulo, SP - 2010

\begin{tabular}{|c|c|}
\hline Características & $\mathbf{N}(\%)$ \\
\hline \multicolumn{2}{|l|}{ Sexo } \\
\hline Masculino & $61(39,6)$ \\
\hline Feminino & $93(60,4)$ \\
\hline Média \pm desvio-padrão & $40,9 \pm 7,8$ \\
\hline \multicolumn{2}{|l|}{ Idade (anos) } \\
\hline 20 I-। 30 & $15(9,7)$ \\
\hline $31 ।-\mid 40$ & $61(39,6)$ \\
\hline $41|-| 50$ & $56(36,6)$ \\
\hline$>50$ & $22(14,1)$ \\
\hline Média \pm desvio padrão & $15,9 \pm 7,8$ \\
\hline \multicolumn{2}{|l|}{ Anos de formado } \\
\hline 01 ।-। 05 & $15(9,7)$ \\
\hline 06 I-। 10 & $30(19,5)$ \\
\hline 11 ।-। 15 & $33(21,4)$ \\
\hline $16|-| 20$ & $36(23,4)$ \\
\hline$>20$ & $40(26,0)$ \\
\hline \multicolumn{2}{|l|}{ Categoria profissional } \\
\hline Enfermeiros & $90(58,4)$ \\
\hline Médicos & $41(26,6)$ \\
\hline Auxiliares de enfermagem & $23(14,9)$ \\
\hline \multicolumn{2}{|l|}{ Carga de trabalho semanal (horas) } \\
\hline $12|-| 24$ & $07(4,5)$ \\
\hline $251-130$ & $14(9,1)$ \\
\hline 31 I-। 40 & $13(8,4)$ \\
\hline 41 I- 50 & $19(12,3)$ \\
\hline 51 ॥-। 60 & $34(22,1)$ \\
\hline$>60$ & $67(43,5)$ \\
\hline Antecedentes pessoais $\mathrm{p} /$ hipertensão arterial & $31(20,1)$ \\
\hline Antecedentes familiares $\mathrm{p} /$ hipertensão arterial & $127(82,5)$ \\
\hline Atualmente realiza outros tratamentos de saúde & $48(31,2)$ \\
\hline \multicolumn{2}{|l|}{ Tipo de tratamento } \\
\hline Depressão & $37(77,1)$ \\
\hline Hipotiroidismo & $08(16,7)$ \\
\hline Obesidade & $03 \quad(6,3)$ \\
\hline Já sofreu acidentes durante $o$ atendimento & $67(43,5)$ \\
\hline Perfurocortante & $22(33,0)$ \\
\hline Tabagismo & $31(20,1)$ \\
\hline Ingestão de bebidas alcoólicas & $73(47,5)$ \\
\hline Atividade Física & $54(35,1)$ \\
\hline Atividade Física & $54(35,1)$ \\
\hline
\end{tabular}


Tabela 2 - Características clínicas e antropométricas dos profissionais que atuam em serviços de atendimento pré-hospitalar - São Paulo, SP - 2010

\begin{tabular}{|c|c|}
\hline Características & $\mathbf{N}(\%)$ \\
\hline \multicolumn{2}{|l|}{ Pressão arterial $(\mathrm{mmHg})$} \\
\hline$<140$ e $<90$ & $103(66,9)$ \\
\hline$\geq 140 / \geq 90$ & $51(33,1)$ \\
\hline \multirow{2}{*}{ Média \pm desvio-padrão } & $125,3 \pm 14,9 / 79,3 \pm 10,8$ \\
\hline & \\
\hline Saudável: $<25$ & $54(34,4)$ \\
\hline Sobrepeso: $25-29,9$ & $66(42,9)$ \\
\hline Obesidade: $\geq 30$ & $35(22,7)$ \\
\hline Média \pm desvio-padrão & $27,4 \pm 4,6$ \\
\hline \multicolumn{2}{|l|}{ Circunferência abdominal $(\mathrm{cm})$} \\
\hline \multirow{2}{*}{\multicolumn{2}{|c|}{$\begin{array}{lcc} & \text { Masculino: } & \text { Feminino: } \\
\text { Normal: } & <94 & <80\end{array}$}} \\
\hline & $46(29,9)$ \\
\hline Risco aumentado: $\quad \geq 94 \quad \geq 80$ & $50(32,5)$ \\
\hline \multirow{2}{*}{$\begin{array}{l}\text { Risco muito aumentado: } \geq 102 \quad \geq 88 \\
\text { Média } \pm \text { desvio-padrão }\end{array}$} & $58(37,7)$ \\
\hline & $91,3 \pm 13,1$ \\
\hline \multicolumn{2}{|l|}{ Glicemia (mg/dL) } \\
\hline Normal: $<110$ & $137(89,0)$ \\
\hline Tolerância diminuída a glicose: $>110$ e $<126$ & $13(8,4)$ \\
\hline Diabetes mellitus: $\geq 126$ & $04(2,6)$ \\
\hline Média \pm desvio-padrão & $89 \pm 13,2$ \\
\hline \multicolumn{2}{|l|}{ Colesterol total (mg/dL) } \\
\hline Ótimo: $<200$ & $99(64,3)$ \\
\hline Limítrofe: 200 І-। 239 & $38(24,7)$ \\
\hline Alto: $\geq 240$ & $17(11,0)$ \\
\hline Média \pm desvio-padrão & $188,8 \pm 37,1$ \\
\hline LDL-c: Low Density Lipoprotein (mg/dL) & \\
\hline Ótimo: $<100$ & $52(33,8)$ \\
\hline Desejável: 100 |ー। 129 & $55(35,7)$ \\
\hline Limítrofe: 130 ।ー। 159 & $35(22,7)$ \\
\hline Alto: 160 |-। 189 & $10(6,5)$ \\
\hline Muito alto: $\geq 190$ & $02(1,3)$ \\
\hline Média \pm desvio-padrão & $116,3 \pm 30,6$ \\
\hline HDL-c: High Density Lipoprotein (mg/dL) & \\
\hline Baixo: $<40$ & $43(27,9)$ \\
\hline Limítrofe: 40 ।- 60 & $94(61,0)$ \\
\hline Alto: $>60$ & $17(11,0)$ \\
\hline Média \pm desvio-padrão & $47,5 \pm 11,9$ \\
\hline Triglicérides (mg/dL) & \\
\hline Ótimo: $<150$ & $108(70,1)$ \\
\hline Limítrofe: 150 a 200 & $21(13,6)$ \\
\hline Alto: 201 a 499 & $23(14,9)$ \\
\hline Muito alto: $\geq 500$ & $02(1,3)$ \\
\hline Média \pm desvio-padrão & $128,1 \pm 85,2$ \\
\hline Proteína $\mathbf{C}$ reativa $(\mathrm{mg} / \mathrm{dL})$ & \\
\hline Primeiro quartil: $<0,1$ & $41(26,6)$ \\
\hline Segundo quartil: $0,1|-| 0,3$ & $51(33,1)$ \\
\hline Terceiro quartil: $0,3-\mid 0,5$ & $38(24,7)$ \\
\hline Quarto quartil: $>0,5$ & $24(15,6)$ \\
\hline Média \pm desvio-padrão: $0,2 \pm 0,8$ & \\
\hline Escore de Risco Framingham & \\
\hline Baixo risco $(\geq 10 \%)$ & $136(88,4)$ \\
\hline Médio Risco $(>10 \%$ e $<20 \%)$ & $16(10,3)$ \\
\hline Alto risco $(\geq 20 \%)$ & $02(1,3)$ \\
\hline
\end{tabular}


Os dados clínicos e antropométricos encontram-se sumarizados na Tabela 2 . Em relação à pressão arterial verificou-se que $33,1 \%$ dos profissionais apresentavam níveis pressóricos considerados alterados, compatíveis com hipertensão arterial; mais da metade estava com seu Índice de Massa Corpórea e cintura abdominal também alterados $(65,6 \%$ e $70,2 \%$, respectivamente). Observou-se que $11 \%$ apresentaram glicemia acima da faixa da normalidade. Os níveis de colesterol estavam acima do desejável em $35,7 \%$, o LDL-c em $29,2 \%$ e triglicérides em $29,8 \%$. Apenas $11,0 \%$ apresentaram níveis ideais de HDL-c e 40,3\% proteína $\mathrm{C}$ reativa nos maiores quartis. Em relação ao Escore de Risco de Framingham, 11,6\% classificaram-se na faixa de médio ou alto risco para apresentarem evento coronariano em 10 anos.

Os dados da Tabela 3 apresentam as variáveis estudadas que se associaram, de forma estatisticamente significante $(p<0,05)$, com os níveis da pressão arterial, classificados ou não em hipertensão. Dessa forma a presença de níveis compatíveis com hipertensão arterial associou-se ao sexo masculino, idade acima de 40 anos, trabalhar frequentemente cansado e o Escore de Risco Framingham nos níveis médio e alto.

Tabela 3 - Distribuição dos profissionais que atuam em serviços de atendimento pré-hospitalar, segundo as variáveis que se associaram com a pressão arterial normal ou alterada - São Paulo, SP - 2010

\begin{tabular}{|c|c|c|c|c|c|}
\hline \multirow{3}{*}{ Variável } & \multicolumn{4}{|c|}{ Pressão Arterial } & \multirow{3}{*}{ Valor de $p$} \\
\hline & $<140$ mmHg e/ou & $<90 \mathrm{mmHg}$ & $\geq 140 \mathrm{mmHg}$ e/ou & $\geq 90 \mathrm{mmHg}$ & \\
\hline & $\mathbf{N}$ & $\%$ & $\mathbf{N}$ & $\%$ & \\
\hline \multicolumn{6}{|l|}{ Sexo } \\
\hline Masculino & 32 & 52,5 & 29 & 47,5 & 0,02 \\
\hline Feminino & 71 & 76,3 & 22 & 23,7 & \\
\hline \multicolumn{6}{|l|}{ Idade (anos) } \\
\hline$\leq 40$ & 56 & 54,6 & 20 & 39,2 & 0,02 \\
\hline$>40$ & 47 & 45,6 & 31 & 60,7 & \\
\hline \multicolumn{6}{|l|}{ Trabalha cansado } \\
\hline Frequentemente & 06 & 35,3 & 11 & 64,7 & \\
\hline Algumas vezes & 54 & 74,0 & 19 & 26,0 & 0,01 \\
\hline Raramente/nunca & 43 & 67,1 & 21 & 32,9 & \\
\hline \multicolumn{6}{|l|}{$\begin{array}{l}\text { Escore de Risco } \\
\text { de Framingham }\end{array}$} \\
\hline Baixo risco $(\leq 10 \%)$ & 98 & 72,0 & 38 & 28,0 & \\
\hline $\begin{array}{l}\text { Médio Risco (>10\% e } \\
<20 \%)\end{array}$ & 05 & 31,0 & 11 & 62,0 & 0,00 \\
\hline Alto risco $(\geq 20 \%)$ & - & - & 02 & 100 & \\
\hline
\end{tabular}

Nos dados da Tabela 4 observa-se a distribuição das variáveis antropométricas e laboratoriais em relação aos níveis pressóricos entre os profissionais que se encontravam nas categorias consideradas alteradas para as variáveis: índice de massa corporal, circunferência abdominal, nível de colesterol total e glicemia apresentaram valores mais elevados da pressão arterial. Já para aqueles com o nível de HDL-c considerado baixo, portanto, inadequado, cursavam com maiores níveis pressóricos sistólicos.
No modelo de regressão logística verificou-se que a presença de hipertensão arterial associou-se com as variáveis HDL-c e Escore de Risco de Framingham. Assim, o HDL-c apareceu como um fator protetor. A chance de apresentar hipertensão diminuiu em, aproximadamente, $25 \%$ quando o HDL-c encontrava-se na faixa acima de 40 $\mathrm{mg} / \mathrm{dL}$. Por outro lado, para o Escore de Risco de Framingham a chance de apresentar hipertensão aumentou 23 vezes quando se muda da faixa de médio para alto risco (Tabela 5). 
Tabela 4 - Distribuição dos profissionais que atuam em serviços de atendimento pré-hospitalar, segundo as variáveis laboratoriais e antropométricas em relação aos níveis da pressão arterial - São Paulo - SP - 2010

\begin{tabular}{|c|c|c|c|c|}
\hline \multirow{3}{*}{ Variável } & \multicolumn{4}{|c|}{ Pressão Arterial Casual } \\
\hline & \multicolumn{2}{|c|}{ Pressão Arterial Sistólica } & \multicolumn{2}{|c|}{ Pressão Arterial Diastólica } \\
\hline & Média \pm DP & Valor de p & Media \pm DP & Valor de $p$ \\
\hline \multicolumn{5}{|l|}{ Índice de Massa Corpórea } \\
\hline Normal & $117,6 \pm 13,1$ & & $75,2 \pm 9,6$ & \\
\hline Sobrepeso & $128,9 \pm 15,9$ & 0,00 & $80,8 \pm 11,9$ & 0,00 \\
\hline Obeso $^{c}$ & $130,2 \pm 10,7$ & & $82,7 \pm 8,4$ & \\
\hline \multicolumn{5}{|l|}{ Circunferência Abdominal } \\
\hline Normal & $119,3 \pm 17,1$ & & $75,1 \pm 11,2$ & \\
\hline Risco aumentado & $126,4 \pm 14,9$ & 0,00 & $80,0 \pm 11,0$ & 0,00 \\
\hline Risco muito aumentado & $129,1 \pm 11,4$ & & $82,1 \pm 9,4$ & \\
\hline \multicolumn{5}{|l|}{ Colesterol total } \\
\hline Ótimo & $122,7 \pm 13,0$ & & $78,3 \pm 9,9$ & \\
\hline Limítrofe & $129,2 \pm 18,7$ & 0,01 & $79,4 \pm 13,1$ & 0,04 \\
\hline Alto & $131,6 \pm 12,5$ & & $85,3 \pm 8,7$ & \\
\hline \multicolumn{5}{|l|}{ LDL-c } \\
\hline Ótimo & $123,2 \pm 14,1$ & & $78,0 \pm 9,8$ & \\
\hline Desejável & $124,1 \pm 12,7$ & & $78,6 \pm 10,8$ & \\
\hline Limítrofe & $126,0 \pm 17,9$ & 0,06 & $79,6 \pm 12,2$ & 0,15 \\
\hline Alto & $137,2 \pm 15,6$ & & $85,9 \pm 5,1$ & \\
\hline Muito alto & $135,5 \pm 22,6$ & & $90,5 \pm 26,1$ & \\
\hline \multicolumn{5}{|l|}{ HDL-c } \\
\hline Baixo & $129,3 \pm 13,7$ & & $81,8 \pm 10,0$ & \\
\hline Limítrofe & $125,0 \pm 15,3$ & 0,01 & $79,1 \pm 11,2$ & 0,06 \\
\hline Alto & $116,9 \pm 12,2$ & & $74,6 \pm 8,9$ & \\
\hline \multicolumn{5}{|l|}{ Triglicérides } \\
\hline Ótimo & $123,4 \pm 14,9$ & \multirow{4}{*}{0,07} & $78,6 \pm 10,6$ & \multirow{4}{*}{0,61} \\
\hline Limítrofe & $128,2 \pm 15,8$ & & $80,6 \pm 10,1$ & \\
\hline Alto & $130,1 \pm 13,1$ & & $81,2 \pm 12,7$ & \\
\hline Muito alto & $140,5 \pm 3,5$ & & $84,0 \pm 1,4$ & \\
\hline \multicolumn{5}{|l|}{ Glicemia } \\
\hline Normal & $124,0 \pm 13,7$ & & $78,3 \pm 10,3$ & \\
\hline Tolerância diminuída glicose & $136,6 \pm 23,0$ & 0,00 & $87,3 \pm 13,3$ & 0,00 \\
\hline Diabetes mellitus & $133,7 \pm 4,5$ & & $88,2 \pm 2,8$ & \\
\hline \multicolumn{5}{|l|}{ Proteína $\mathrm{C}$ reativa $(\mathrm{mg} / \mathrm{dL})$} \\
\hline Primeiro quartil & $122,2 \pm 16,6$ & & $76,0 \pm 11,0$ & \\
\hline Segundo quartil & $127,5 \pm 13,0$ & 023 & $79,3 \pm 10,1$ & \\
\hline Terceiro quartil & $123,7 \pm 15,0$ & 0,23 & $80,5 \pm 11,4$ & 0,10 \\
\hline Quarto quartil & $128,4 \pm 15,1$ & & $83,1 \pm 10,0$ & \\
\hline
\end{tabular}

Tabela 5 - Resultados do modelo de regressão logística para presença de hipertensão arterial, de acordo com a medida casual da pressão arterial dos profissionais que atuam em serviços de atendimento pré-hospitalar - São Paulo - SP - 2010

\begin{tabular}{lcccccc}
\hline \multicolumn{1}{c}{ Variável } & Parâmetro estimado & Erro padrão & $\mathbf{p}$ & Odds Ratio & & IC 95\% \\
Intercepto & $-0,683$ & 0,177 & 0,00 & & & \\
HDL-c $>\mathbf{4 0} \mathbf{~} \mathbf{~ g / d L}$ & $-1,360$ & 0,589 & 0,02 & 0,257 & 0,081 & 0,813 \\
Escore de Risco de Framingham $\mathbf{> 1 0} \%$ & 3,142 & 1,242 & 0,01 & 23,159 & 2,029 & 264,378 \\
\hline
\end{tabular}




\section{DISCUSSÃO}

O presente estudo identificou uma população relativamente jovem em plena atividade laboral com elevada prevalência de fatores de risco cardiovascular. A prevalência de $33,1 \%$ para a hipertensão arterial entre os profissionais de saúde estudados é um achado coincidente com estudos nacionais. Considera-se que, no Brasil, $30 \%$ dos adultos sejam hipertensos podendo dobrar na faixa etária dos idosos ${ }^{(6)}$. Estudos realizados entre outros grupos específicos de trabalhadores demonstraram uma prevalência variável de hipertensão arterial. Em trabalhadores da indústria de refrigerantes ${ }^{(9)}$ observou-se $27 \%$ de hipertensos e em trabalhadores de empresa metalúrgica e siderúrgica $24,7 \%^{(10)}$. Em funcionários de um complexo hospitalar da cidade de São Paulo a prevalência foi de $26 \%^{(11)}$ e, também na cidade de São Paulo, estudo com prevalência referida mostrou índice de $32 \%{ }^{(12)}$.

A prevalência de hipertensão do presente estudo pode ter sido influenciada pelo tipo de atividade realizada por esses profissionais, como a exposição mais intensa ao estresse físico e mental e alteração do ritmo circadiano durante a jornada de trabalho (geralmente de 24 horas). Tem-se verificado estreita relação entre estresse e elevação dos níveis pressóricos ${ }^{(13)}$.

Outro aspecto que poderia influenciar a prevalência de hipertensão observada foi o achado do índice de massa corporal acima dos níveis considerados saudáveis em $65,6 \%$ dos profissionais. Tal achado supera os dados de um estudo ${ }^{(14)}$ que observou uma proporção de sobrepeso de $35,7 \%$ e obesidade de $17,7 \%$, entre profissionais de saúde da cidade de Teresina no Piauí. Investigação com motoristas de caminhão que trafegavam pela Rodovia Regis Bittencourt, no trecho paulista, mostrou índices também elevados, pois $46 \%$ tinham sobrepeso, $36 \%$ obesidade e $58 \%$ circunferência abdominal aumentada ${ }^{(15)}$. O presente estudo mostrou ainda que o sobrepeso esteve mais presente no sexo masculino, e a obesidade no sexo feminino.

Os dados da literatura demonstram a importância do excesso de peso na hipertensão arterial. O estudo de Framingham relatou que $70 \%$ de novos casos de hipertensão arterial estavam relacionados ao excesso de massa corpórea ${ }^{(16)}$. Dada à associação entre hipertensão, envelhecimento e obesidade, pode-se supor que a prevalência de hipertensão entre os profissionais estudados possa progressivamente aumentar se estes não alterarem seu estilo de vida e controlarem o peso corpóreo, já que o envelhecimento é uma variável não modificável. Estudo com hipertensos atendidos em uma Liga de Hipertensão na cidade de São Paulo mostrou que pressão arterial mais elevada (diastólica $>110 \mathrm{mmHg}$ ) se associou com envelhecimento e aumento de peso corporal $^{(17)}$. Concomitante aos altos níveis de sobrepeso e obesidade verificou-se ainda que $70 \%$ dos profissionais apresentavam cintura abdominal acima do considerado normal, dados estes que preocupam. Neste contexto destaca-se em relação às características do tecido subcutâneo abdominal, que ele constitui-se em um compartimento do tecido adiposo de grande influência na sensibilidade à insulina predispondo ao Diabetes mellitus, além de secretar mediadores inflamatórios e, assim, aumentando o risco para Diabetes mellitus e intolerância à glicose. A esse cenário acrescenta-se o elevado índice de sedentarismo, pois $65 \%$ dos participantes não realizavam nenhuma atividade física. É amplamente aceito que a atividade física regular conduz a uma série de respostas benéficas do sistema cardiovascular. $\mathrm{Na}$ população estudada surpreende a alta prevalência de sedentarismo, já que esses profissionais estão expostos, constantemente, a demandas físicas em suas atividades rotineiras no pré-hospitalar.

Em relação ao perfil lipídico os achados também foram preocupantes. Têm se evidenciado relação positiva entre o excesso de peso e a dislipidemia, sendo encontradas elevações leves à moderadas dos níveis séricos de triglicérides e níveis séricos mais baixos de HDL-c. Contudo, os níveis séricos de LDL-c podem ou não estar aumentados( ${ }^{(6)}$. A dislipidemia está envolvida na gênese da aterosclerose que se caracteriza por uma resposta inflamatória da parede vascular, as injúrias, como o aumento do LDL-c e proteína $C$ reativa. No presente estudo o achado de $15 \%$ dos profissionais com altos níveis de proteína $C$ reativa pode caracterizar um estado de inflamação e/ou infecção. A relação entre o nível de HDL-c e pressão arterial demonstra a sua importância, tendo em vista que permaneceu no modelo de regressão logística.

Outro aspecto relevante foi a presença do tabagismo e ingestão de bebidas alcoólicas, com dados superiores a achados de estudos. Observa-se que $47,5 \%$ dos profissionais referiu uso de bebida alcoólica, índice superior ao do estudo ${ }^{(18)} \mathrm{em}$ população geral da área rural de Minas Gerais (37,6\%).

A referência de outros problemas de saúde foi expressiva, pois cerca de um quarto dos profissionais citou tratar a depressão. Dessa forma, a prevalência de depressão nesse grupo foi maior do que na população geral, cuja prevalência estimada é em torno de $3 \%$ a $11 \%^{(18)}$ e maior também à encontrada em residentes de enfermagem na pesquisa ${ }^{(19)}$ que, utilizando o Índice de Depressão de Beck, verificou uma prevalência de 19,1\%. Em nosso meio estudo com amostra de 258 motoristas profissionais de transporte de cargas em uma rodovia brasileira encontrou prevalência de 37\% de hipertensão e $33 \%$ eram portadores de possíveis transtornos mentais comuns e os participantes referiram sentirem-se nervosos, tensos ou preocupados (56\%), dormirem mal (47\%), dores de cabeça (37\%), ter dificuldade de tomar decisões $(38 \%)$ e dificuldade de pensar com clareza $(20 \%)^{(20)}$. A depressão é uma doença altamente incapacitante e crônica. Além dos sintomas emocionais, cognitivos e físicos, exerce importante influência na qualidade de vida. Den- 
tre os sintomas da depressão, ocorre humor deprimido, perda do interesse, fadigabilidade, redução da concentração e atenção, sono perturbador, apetite diminuído ou aumentado. Nesse grupo de profissionais a diminuição da concentração e atenção, em especial, é extremamente perigosa, pois estão expostos em situação de risco biológico e ambiental extremos, podendo aumentar as chances de acidentes de trabalho. Quase metade dos profissionais $(43,5 \%)$ referiu acidente de trabalho e, aproximadamente um terço deles (33\%) foi acidente com material perfurocortante.

Analisando-se a estratificação de risco cardiovascular da amostra estudada e dentro do conceito atual de prevenção cardiovascular, quando aplicado o ERF, a maioria expressiva $(88,4 \%)$ encontrava-se na faixa de baixo risco, dados semelhantes aos de pesquisadores ${ }^{(21)}$ em sua amostra entre motoristas de ônibus (85\%). A presença de maior prevalência de pessoas no escore de baixo risco provavelmente deva-se ao fato de se encontrarem na quarta década de vida, e a idade é um fator importante na determinação do ERF. Deve-se destacar que o princípio fundamental da prevenção é de que um grande número de indivíduos expostos a um risco baixo, provavelmente produzirá mais casos do que um pequeno número de pessoas expostas a um alto risco. O ERF demonstrou ser uma ferramenta importante como preditor de doença coronariana, pois permaneceu no modelo de regressão logística.

\section{REFERÊNCIAS}

1. World Health Organization (WHO). Who Statistical Information System [Internet]. Geneva; 2010 [cited 2010 Sept 8]. Available from: http://www.who.int/whosis/database/

2. Brasil. Ministério da Saúde. Vigitel Brasil 2009: vigilância de fatores de risco e proteção para doenças crônicas por inquérito telefônico [Internet]. Brasília; 2010 [citado 2011 maio 15]. Disponível em: http://portal.saude. gov.br/portal/arquivos/pdf/vigitel_2009_preliminar_ web_20_8_10.pdf

3. Mancia G, Grassi G. The new European Society of Hypertension/European Society of Cardiology (ESH/ESC) Guidelines. Ther Adv Cardiovasc Dis. 2008;2(1):5-12.

4. KotsisV, Stabouli S, Bouldin M, Low A, Toumanidis S, Zakopoulos N. Impact of obesity on 24-hour ambulatory blood pressure and hypertension. Hypertension. 2005;45(2):602-7.

5. Polanczyc CA. Fatores de risco cardiovascular no Brasil: os próximos 50 anos. Arq Bras Cardiol. 2005;84(3):199-201.

\section{CONCLUSÃO}

Os achados do presente estudo mostraram elevada prevalência de hipertensão arterial e forte associação com ERF. Os dados chamam a atenção por se tratar, principalmente, de profissionais da área da saúde com faixa etária relativamente jovem. A presença de estilos e hábitos de vida inadequados, aliados às características do trabalho da amostra estudada, foram determinantes importantes para a presença de níveis da pressão arterial compativeis com hipertensão arterial. Salienta-se que estudos transversais não permitem a possibilidade de atribuir causalidade às associações encontradas, já que analisam desfecho e exposição simultaneamente, podendo ser uma das limitações do presente estudo. Entretanto, verificou-se que os profissionais estudados estão expostos a risco cardiovascular, em especial os resultantes do aumento do índice de massa corporal. O desafio é ainda maior por se tratar de profissionais da área da saúde, grupo que detém a informação. Deve ser estimulada a conciliação entre a experiência profissional, adquirida ao longo dos anos de atuação no serviço pré-hospitalar, com boas condições de saúde através da prática de atividade física, como forma de prevenção e reversão dos altos níveis de sobrepeso, obesidade e sedentarismo. Por outro lado, cabe às instituições seu papel de zelar por boas condições de saúde de seus trabalhadores. A excessiva jornada de trabalho identificada, provavelmente seja decorrente de salários insuficientes para garantir uma renda mínima satisfatória.

6. Sociedade Brasileira de Hipertensão; Sociedade Brasileira de Cardiologia; Sociedade Brasileira de Nefrologia. VI Diretrizes Brasileiras de Hipertensão Arterial. Rev Bras Hipertensão. 2010;17(1):1-69.

7. Organização Mundial da Saúde (OMS). Obesidade: prevenindo e controlando a epidemia global. São Paulo: Roca; 2004.

8. Wilson PWF, Agostino RBD, Levy D, Belangen AM, Silbershat $\mathrm{H}$, Kannel WB. Prediction of coronary heart disease using risk factor categories. Circulation. 1998;97(18):1837-47.

9. Cassani RSL, Nobre F, Pazin Filho A, Schmidt A. Prevalência de fatores de risco cardiovascular em trabalhadores de uma indústria brasileira. Arq Bras Cardiol. 2009;92(1):16-22.

10. Martinez MC, Latorre MRDO. Fatores de risco para hipertensão arterial e diabete mellitus em trabalhadores de empresa metalúrgica e siderúrgica. Arq Bras Cardiol. 2006;87(4):471-79.

11. Mion Junior D, Pierin AMG, Bambirra AP, Assunção JH, Monteiro JM, Chinen RY, et al. Hypertension in employees of a university general hospital. Rev Hosp Clin Fac Med S Paulo. 2004;59(6):329-36.

$\begin{array}{rr}\text { Rev Esc Enferm USP } & \text { Risco cardiovascular em profissionais de saúde } \\ 2012 ; 46(2): 395-403 & \text { de serviços de atendimento pré-hospitalar }\end{array}$


12. Mion Junior D, Pierin AMG, Bensenor IM, Marin JCM, Costa KRA, Henrique LFO, et al. Hypertension in the city of São Paulo: self-reported prevalence assessed by telephone surveys. Arq Bras Cardiol. 2010;95(1):99-106.

13. Rau R. The association between blood pressure and work stress: the importance of measuring isolated systolic hypertension. Work Stress. 2006;20(1):84-97.

14. Sousa RMRP, Sobral DP, Paz SMRS, Martins MCC. Prevalência de sobrepeso e obesidade entre funcionários plantonistas de Unidades de Saúde de Teresina, Piauí - BR. Rev Nutr. (Campinas). 2007;20(5):473-82.

15. Cavagioni LC, Pierin AMG. Hipertensão arterial e obesidade em motoristas profissionais de transporte de cargas. Acta Paul Enferm. 2010;23(4)455-60.

16. Garrison RJ, Kannel WB, Stokes J, Castelli WP. Incidence and precursors of hypertension in young adults: the Framingham Offspring Study. Prev Med. 1987;16(2):235-51.
17. Pierin AMG, Mion Junior D, Fukushima JT, Pinto AR, Kaminaga MM. The profile of hypertensive patients related to awareness and level of hypertension. Rev Esc Enferm USP. 2001;35(1):11-8.

18. Fleck MPA, Lafer B, Sougey EB, Del Porto JA, Brasil MA, Juruena MF. Diretrizes da Associação Médica Brasileira para o tratamento da depressão. Rev Bras Psiquiatr. 2003;25(2):114-22.

19. Franco GP, Barros ALBL, Nogueira-Martins LA. Qualidade vida e sintomas depressivos em residentes de enfermagem. Rev Latino Am Enferm. 2005;13(2):139-44.

20. Cavagioni LC, Pierin AMG, Batista KM, Bianchi ERF, Costa ALS. Health problems, hypertension and predisposition to stress in truck drivers. Rev Esc Enferm USP [Internet]. 2009 [cited 2011 Apr 12];43(n.esp 2):1267-71. Available from: http://www.scielo.br/pdf/reeusp/v43nspe2/en_a21v43s2.pdf

21. Landim MBP, Victor EG. Framingham score for public transportation drivers in the city of Teresina, Piauí. Arq Bras Cardiol. 2006;87(3):280-5. 